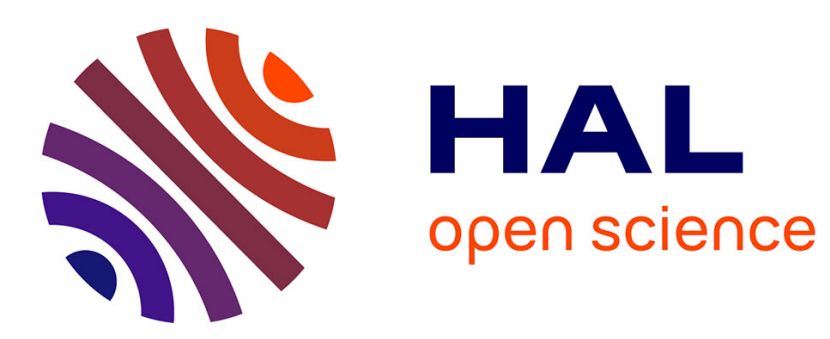

\title{
Impact of Noise Correlation on Multimodality
}

Saloua Chlaily, Pierre-Olivier Amblard, Olivier J.J. Michel, Christian Jutten

\section{To cite this version:}

Saloua Chlaily, Pierre-Olivier Amblard, Olivier J.J. Michel, Christian Jutten. Impact of Noise Correlation on Multimodality. EUSIPCO 2016 - 24th European Signal Processing Conference, Aug 2016, Budapest, Hungary. hal-01382832

\section{HAL Id: hal-01382832 \\ https://hal.science/hal-01382832}

Submitted on 17 Oct 2016

HAL is a multi-disciplinary open access archive for the deposit and dissemination of scientific research documents, whether they are published or not. The documents may come from teaching and research institutions in France or abroad, or from public or private research centers.
L'archive ouverte pluridisciplinaire HAL, est destinée au dépôt et à la diffusion de documents scientifiques de niveau recherche, publiés ou non, émanant des établissements d'enseignement et de recherche français ou étrangers, des laboratoires publics ou privés. 


\title{
Impact of Noise Correlation on Multimodality
}

\author{
Saloua Chlaily, Pierre-Olivier Amblard, Olivier Michel and Christian Jutten \\ Gipsa-Lab, CNRS, Université Grenoble Alpes, France
}

\begin{abstract}
In this paper, we consider the problem of estimating an unknown random scalar observed by two modalities. We study two scenarios using mutual information and mean square error. In the first scenario, we consider that the noise correlation is known and examine its impact on the information content of two modalities. In the second scenario we quantify the information loss when the considered value of the noise correlation is wrong. It is shown that the noise correlation usually enhances the estimation accuracy and increases information. However, the performance declines if the noise correlation is misdefined, and the two modalities may jointly convey less information than one single modality.
\end{abstract}

\section{INTRODUCTION}

Recently, in various domains, different sensors i.e. modalities are used simultaneously to observe several aspects of the phenomenon studied, hopefully ensuring a better understanding: this concept is called Multimodality. Multimodality exploits the links between modalities, to enable their interaction and therefore reveal more information about the phenomenon than using a unique modality. However, the expected improvement is not always observed. That is because multimodality introduces new challenges: different resolutions, sizes, missing data, inconsistency, to name a few, and noise. Noise is one of the challenges rarely addressed since most studies ignore it or consider independent noises [1]. However, the correlation between noises, if it exists, represent an important link that must be considered for ensuring better performance.

The use of different modalities is important in many applications where phenomena are extremely complex (e.g. neuroimaging, human-computer interface, remote sensing, etc). Furthermore, the benefit of using different modalities is very application dependent. Important challenges in multimodal signal processing are not only to process jointly several modalities, but also to a priori quantify the benefits (if any) of multimodal integration. The work presented here is a small step in this direction. To draw conclusions independently of the applications, we consider a model which is simple to analyze, easy to interpret, but that yet provides interesting conclusions about the effect of interaction between modalities onto inference tasks.

The model involves only two modalities and a few parameters. Considering each modality as a communication channel, we address the following questions in terms of mutual information and of minimum mean square error (MMSE).

1) Under which conditions, a single modality carry more information than two modalities?

This work is supported by the project CHESS, 2012-ERC-AdG-320684.
2) What is the importance of noise correlation on inference accuracy?

3) What is the effect of misdefining the links between noises on the information content of modalities and inference accuracy?

The model considered in this work, was studied in [2] in terms of Fisher information, while still under the exact model assumption. The paper is organized as follows. The proposed model and main notations are presented in Section II. In Section III, we recall some notions of information theory. The impact of the links between modalities on the information content is presented in Section IV. In Section V we investigate the effect of misdefining the links between modalities. Finally, the conclusions and perspectives are drawn in Section VI.

\section{NOTATIONS AND MODEL}

Let $X_{1}$ and $X_{2}$ be two noisy observations of a random scalar parameter $S$. For simplicity, we ignore the sensor response:

$$
\left\{\begin{array}{l}
X_{1}=S+B_{1} \\
X_{2}=S+B_{2}
\end{array}\right.
$$

where $S$ and the noises $B_{1}$ and $B_{2}$ are assumed to be jointly Gaussian, with zero means and variances $\sigma^{2}, \sigma_{1}^{2}$, and $\sigma_{2}^{2}$, respectively. $S$ is independent of the noises. We denote by $\rho=\frac{E\left[B_{1} B_{2}\right]}{\sigma_{1} \sigma_{2}}$ the noise correlation coefficient, and by $R_{i}=\frac{\sigma^{2}}{\sigma_{i}^{2}}$ the signal to noise ratio (SNR) of modality $X_{i}$. We assume without loss of generality that $R_{1} \geq R_{2}$, unless otherwise stated.

\section{UNCERTAINTY OF EXACT AND WRONG MODELS}

In this section, we introduce the differential entropy (DE) and the mutual information (MI) [3], which we will use in the next sections for studying the model (1).

The DE quantifies the uncertainty of a continuous random variable, i.e. the information that would be obtained by observing this variable. Let $X$ be a continuous random variable with a probability density function (pdf) $f_{X}$, the DE of $X$ denoted by $H(X)$ is given by:

$$
H(X)=\int f_{X}(x) \log \frac{1}{f_{X}(x)} d x .
$$

Eq. (2) assumes that the pdf $f_{X}$ is known. In fact, if we do not know the exact probability of $X$, and assume a wrong pdf $\hat{f}_{X}$ instead of $f_{X}$, the measure of Shannon information is function of $\hat{f}_{X}$, while the average is over the exact probability:

$$
H_{\hat{f}_{X} \mid f_{X}}(X)=\int f_{X}(x) \log \frac{1}{\hat{f}_{X}(x)} d x .
$$


The notation $H_{\hat{f}_{X} \mid f_{X}}(X)$ denotes the expected uncertainty of the random variable $X$ when we believe that its pdf is $\hat{f}_{X}$ while it is $f_{X}$. In coding theory, for discrete variables, $H_{\hat{f}_{X} \mid f_{X}}(X)$ is the number of bits needed to code an event when its probability density function is wrongly estimated. If we write $H_{\hat{f}_{X} \mid f_{X}}$ as a function of $H$, we find:

$$
H_{\hat{f}_{X} \mid f_{X}}(X)=H(X)+D_{K L}\left(f_{X}(X) \| \hat{f}_{X}(X)\right),
$$

where $D_{K L}\left(f_{X}(X) \| \hat{f}_{X}(X)\right)=\int f_{X}(x) \log \frac{f_{X}(x)}{\hat{f}_{X}(x)} d x$ is the Kullback-Leibler (KL) divergence between the densities $f_{X}$ and $\hat{f}_{X}$. Since $D_{K L}$ is always nonnegative and is zero iff $\hat{f}_{X}=f_{X}$ [3], (4) leads to the following interpretation: when we assume $\hat{f}_{X} \neq f_{X}$, the uncertainty of $X$ increases and the error $H_{\hat{f}_{X} \mid f_{X}}-H$ is given by the KL divergence between exact and wrong densities. Clearly, the error on the uncertainty increases with the KL divergence i.e with the pdf mismatch.

Let $Y$ and $Z$ be two random variables linked to a third $X$. Observing $Y$ and $Z$ reduces the uncertainty of $X$. The amount of information that $Y$ and $Z$ reveal jointly about $X$ is given by the MI:

$$
\begin{aligned}
I(X ;(Y, Z)) & =H(X)-H(X \mid Y, Z) \\
& =D_{K L}\left(f_{X Y Z}(x, y, z) \| f_{X}(x) f_{Y Z}(y, z)\right),
\end{aligned}
$$

where $H(X \mid Y, Z)=-E_{f_{X Y Z}} \log f_{X \mid Y Z}(x \mid y, z)$ is the conditional DE that quantifies the remaining uncertainty of $X$ given $(Y, Z) \cdot f_{X \mid Y Z}(x \mid y, z)$ is the conditional pdf of the random vector $X$ given $(Y, Z) . f_{X Y Z}(x, y, z)$ and $f_{Y Z}(y, z)$ are the joint densities for $(X, Y, Z)$ and $(Y, Z)$ respectively. If we consider a wrong conditional pdf for $(Y, Z)$ given $X$, $\hat{f}_{Y Z \mid X}(y, z \mid x)$, the amount of information revealed jointly by $Y$ and $Z$ about $X$ would be wrongly evaluated as:

$$
\begin{aligned}
& I_{\hat{f}_{Y Z \mid X} \mid f_{Y Z \mid X}}(X ;(Y, Z)) \\
& =H(X)-H_{\hat{f}_{Y Z \mid X} \mid f_{Y Z \mid X}}(X \mid Y, Z) \\
& =I(X ;(Y, Z))-D_{K L}\left(f_{X \mid Y Z}(x \mid y, z) \| \hat{f}_{X \mid Y Z}(x \mid y, z)\right)
\end{aligned}
$$

where:

and

$$
\hat{f}_{X \mid Y Z}(x \mid y, z)=\frac{\hat{f}_{Y Z \mid X}(y, z \mid x) f_{X}(x)}{\hat{f}_{Y Z}(y, z)}
$$

$f_{Y Z}(y, z)=\int \hat{f}_{Y Z \mid X}(y, z \mid x) f_{X}(x) d x$

Clearly (6) shows that the MI decreases when we assume a wrong model, due to the property of $D_{K L}$, and the equality $I_{\hat{f}_{Y Z \mid X} \mid f_{Y Z \mid X}}=I$ holds iff $\hat{f}_{Y Z \mid X}=f_{Y Z \mid X}$. Note that $I_{\hat{f}_{Y Z \mid X} \mid f_{Y Z \mid X}}(X ;(Y, Z))$ is not a mutual information, in the sense that it has not the properties of MI. In fact, MI is a KL divergence (nonnegative), while $I_{\hat{f}_{Y Z \mid X} \mid f_{Y Z \mid X}}$ is a difference of KL divergences and it can be negative (cf. Section V).

\section{EXACT MODEL}

In this section we assume that the link between noises is perfectly known, i.e the exact value of the noise correlation coefficient $\rho$ is known. The modalities in (1) can be considered

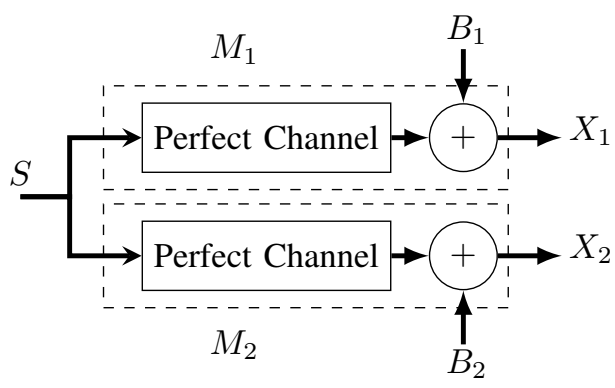

Fig. 1. Modalities as two communication channels

as two noisy communication channels (Fig. 1), where $S$ is the input and $X_{1}, X_{2}$ are the outputs.

Using the Gaussian assumption (Section II), the information carried by the unique modality $X_{i}(i=1,2)$ about $S$ is

$$
I\left(S ; X_{i}\right)=\frac{1}{2} \log \left(1+R_{i}\right) .
$$

Following the assumption $R_{1} \geq R_{2}$ i.e. $X_{1}$ is more reliable than $X_{2}, X_{1}$ provides more information about $S$, i.e. $I\left(S ; X_{1}\right) \geq I\left(S ; X_{2}\right)$. If the modalities are considered jointly, i.e by exploiting their link $\rho$, the information they provide about $S$ is given by (5), and in the Gaussian case it is given by:

$$
\begin{aligned}
I(S & \left.;\left(X_{1}, X_{2}\right)\right) \\
\quad & =\frac{1}{2} \log \left(\frac{\left(1+R_{1}\right)\left(1+R_{2}\right)-\left(\sqrt{R_{1} R_{2}}+\rho\right)^{2}}{1-\rho^{2}}\right) .
\end{aligned}
$$

Eq. (8) depends on the SNRs and on the parameter $\rho$ which summarizes the relation between the two modalities. Thus, in the following we add $\rho$ as a subscript to distinguish between the MI for different values of $\rho$. The notation $I_{\rho}\left(S ;\left(X_{1}, X_{2}\right)\right)$ denotes the MI between $S$ and the couple of modalities $\left(X_{1}, X_{2}\right)$ when the noise correlation coefficient is $\rho$. In Figure 2, we draw $I_{\rho}\left(S ;\left(X_{1}, X_{2}\right)\right)$ (plain), $I_{0}\left(S ;\left(X_{1}, X_{2}\right)\right)$ (dashed) and $I\left(S, X_{1}\right)$ (dotted) as a functions of $\rho$ for equal and different SNRs (Fig. 2a and 2b, respectively), while the interpretations are presented in the following subsections.

From an estimation theory perspective, the optimal MMSE estimator [4] of $S$ as a function of $X_{1}$ and $X_{2}$ is:

$$
\hat{S}_{\rho}\left(X_{1}, X_{2}\right)=\frac{\left(R_{1}-\sqrt{R_{1} R_{2}} \rho\right) X_{1}+\left(R_{2}-\sqrt{R_{1} R_{2}} \rho\right) X_{2}}{\left(1+R_{1}\right)\left(1+R_{2}\right)-\left(\sqrt{R_{1} R_{2}}+\rho\right)^{2}} .
$$

Fig. 3 shows the mean square errors (MSE) of estimations using only one modality, $E\left[\left(S-\hat{S}\left(X_{1}\right)\right)^{2}\right]$ (dotted), and the two modalities $E\left[\left(S-\hat{S}_{\rho}\left(X_{1}, X_{2}\right)\right)^{2}\right]$ (plain) vs. the noise correlation coefficient $\rho$. The interpretations are given in the following subsections while the additional dashed line, $E\left[\left(S-\hat{S}_{0 \mid \rho}\left(X_{1}, X_{2}\right)\right)^{2}\right]$ is discussed in Section V. 


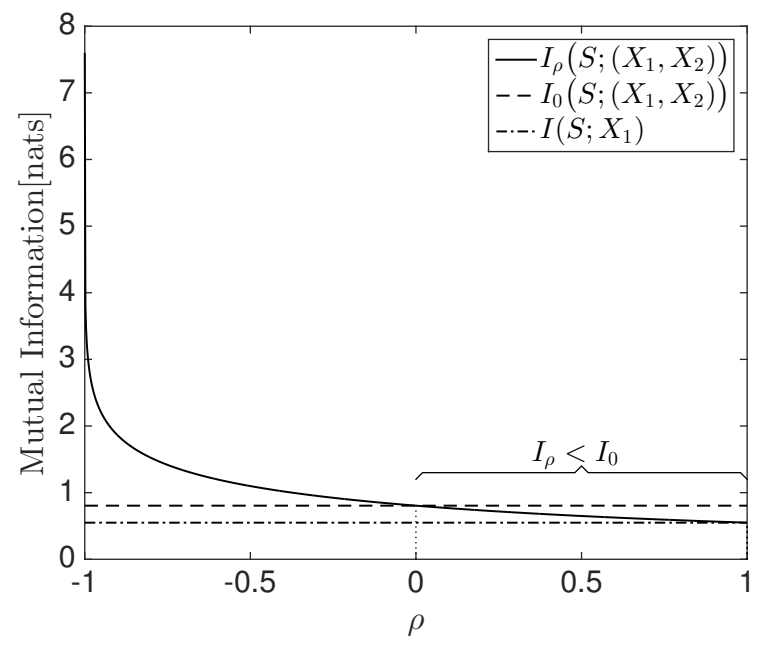

(a) Equal SNRs

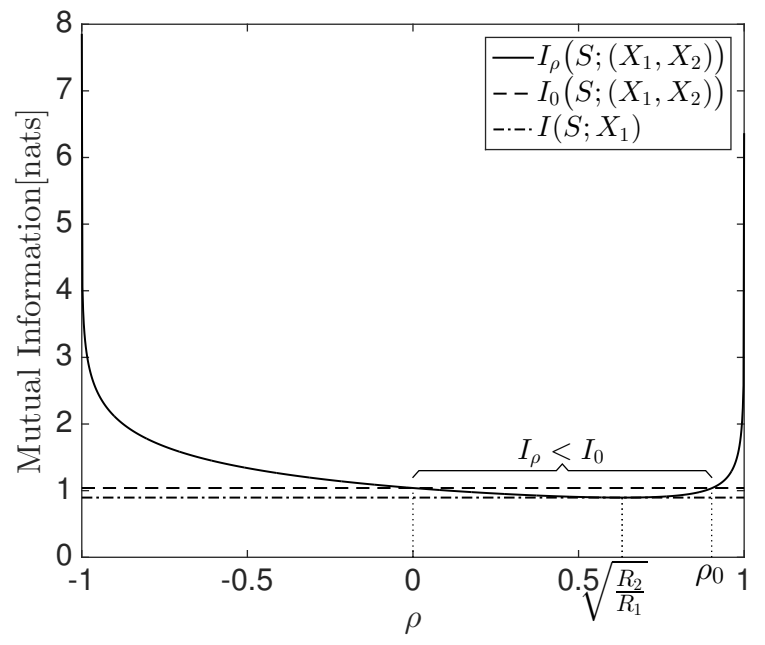

(b) Different SNRs

Fig. 2. The mutual information $I_{\rho}\left(S ;\left(X_{1}, X_{2}\right)\right)$ (plain), $I_{0}\left(S ;\left(X_{1}, X_{2}\right)\right)$ (dashed) and $I\left(S ; X_{1}\right)$ (dotted) as a functions of $\rho$ (known)

\section{A. One or two modalities}

First we would like to know when it is advantageous to combine two modalities. In this purpose, we compare the information brought jointly by the modalities with the information conveyed by a single modality:

$$
I_{\rho}\left(S ;\left(X_{1}, X_{2}\right)\right)-I\left(S ; X_{i}\right)=I_{\rho}\left(S ; X_{j} \mid X_{i}\right), i \neq j
$$

where $I_{\rho}\left(S ; X_{j} \mid X_{i}\right)=H\left(S \mid X_{j}\right)-H\left(S \mid X_{i}, X_{j}\right)$ is the conditional MI that quantifies the additional information carried by $X_{j}$ given $X_{i}$. The conditional MI is always nonnegative, and it equals zero when no further information can be retrieved from the new modality $X_{j}$.

For model (1), from equations (7) and (8), the conditional MI writes:

$$
\begin{aligned}
I_{\rho}(S & \left.; X_{i} \mid X_{j}\right) \\
& =\frac{1}{2} \log \left(\frac{\left(1+R_{1}\right)\left(1+R_{2}\right)-\left(\sqrt{R_{1} R_{2}}+\rho\right)^{2}}{\left(1-\rho^{2}\right)\left(1+R_{j}\right)}\right)
\end{aligned}
$$

$I_{\rho}\left(S ; X_{1} \mid X_{2}\right) \neq 0$ since $X_{1}$ provides more information than $X_{2}$ about $S\left(R_{1}>R_{2}\right)$. On the contrary, the conditional MI $I_{\rho}\left(S ; X_{2} \mid X_{1}\right)$ can vanish for $\rho=\sqrt{\frac{R_{2}}{R_{1}}}$, which means that $X_{2}$ does not provide any new information about $S$, i.e. $X_{2}$ is totally redundant given $X_{1}$. We observe in Fig. $2 \mathrm{~b}$ that the MI related to $X_{1}$ (dotted) is tangent to the MI related to two modalities (plain) for $\rho=\sqrt{\frac{R_{2}}{R_{1}}}$, where the latter attains its minimum. In this case the optimal estimator of $S$ upon $X_{1}$ and $X_{2}, \hat{S}_{\rho}\left(X_{1}, X_{2}\right)$, equals the optimal estimator based only on $X_{1}, \hat{S}\left(X_{1}\right)$ and the MMSE is maximum (Fig. 3b). Therefore, when $\rho=\sqrt{\frac{R_{2}}{R_{1}}}$ it is sufficient to use the modality $X_{1}$ alone. Otherwise, under the exact model assumption it is always beneficial to consider two modalities. Note that, for equal SNRs, i.e. $R_{1}=R_{2}$, at $\rho=1$, the two modalities are completely redundant since $X_{1}=X_{2}$ (Fig. 2a and 3a).

\section{B. Independent or correlated noises}

In the previous subsection, we show that usually the MI related to two modalities is larger than the MI related to a unique modality. In this subsection, we wonder if the existence of links (correlation $\rho$ ) always guarantee higher MI. To this end, we compare the amount of information carried by both modalities when the link between noises exists $(\rho \neq 0), I_{\rho}\left(S ;\left(X_{1}, X_{2}\right)\right)$ and when this link is inexistent $(\rho=0), I_{0}\left(S ;\left(X_{1}, X_{2}\right)\right)$. Following the Gaussian assumption, independence is equivalent to $\rho=0$. Note that modalities with independent noises are not totally independent but conditionally independent given $S$. Using (10), we can write:

$$
\begin{aligned}
I_{\rho}\left(S ;\left(X_{1}, X_{2}\right)\right) & -I_{0}\left(S ;\left(X_{1}, X_{2}\right)\right) \\
& =I_{\rho}\left(S ; X_{2} \mid X_{1}\right)-I_{0}\left(S ; X_{2} \mid X_{1}\right)
\end{aligned}
$$

Eq (12) shows that modalities with independent noises outperform the ones with correlated noises when $I_{\rho}\left(S ; X_{2}\right.$ $\left.X_{1}\right)<I_{0}\left(S ; X_{2} \mid X_{1}\right)$. In Fig. $2 \mathrm{~b}$ we can see that this occurs if $\rho \in] 0, \rho_{0}=\frac{2 \sqrt{R_{1} R_{2}}}{R_{1}+R_{2}}[$, for which the noise correlation induces redundancy. When $R_{2}$ tends towards $R_{1}$, the modalities become more redundant and $\rho_{0}$ tends towards 1 (Fig. 2a). Conversely, when $R_{2}$ is negligible w.r.t $R_{1}, \rho_{0}$ tends to 0 . Beyond this interval i.e $\rho \in[-1,0] \cup\left[\rho_{0}, 1\right]$, $I_{\rho}\left(S ; X_{2} \mid X_{1}\right) \geq I_{0}\left(S ; X_{2} \mid X_{1}\right)$ since redundancy is exploited to enhance the estimation accuracy. In fact, the noise is reduced by summing the two modalities with proper scaling. In particular, $I_{\rho}$ is infinite for $\rho=1\left(R_{1} \neq R_{2}\right)$ and $\rho=-1$ (Fig. 2b), since the noise is completely cancelled and $S$ can be perfectly recovered $(\mathrm{MMSE}=0$, Fig. $3 \mathrm{~b})$ using respectively the optimal estimators (9):

$$
\hat{S}_{1}\left(X_{1}, X_{2}\right)=\frac{\sqrt{R_{1}} X_{1}-\sqrt{R_{2}} X_{2}}{\sqrt{R_{1}}-\sqrt{R_{2}}} .
$$




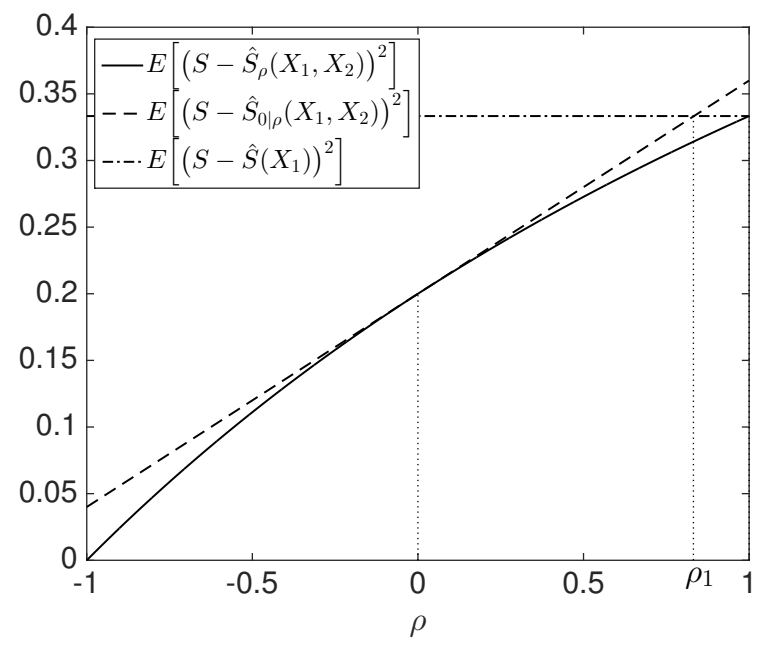

(a) Equal SNRs

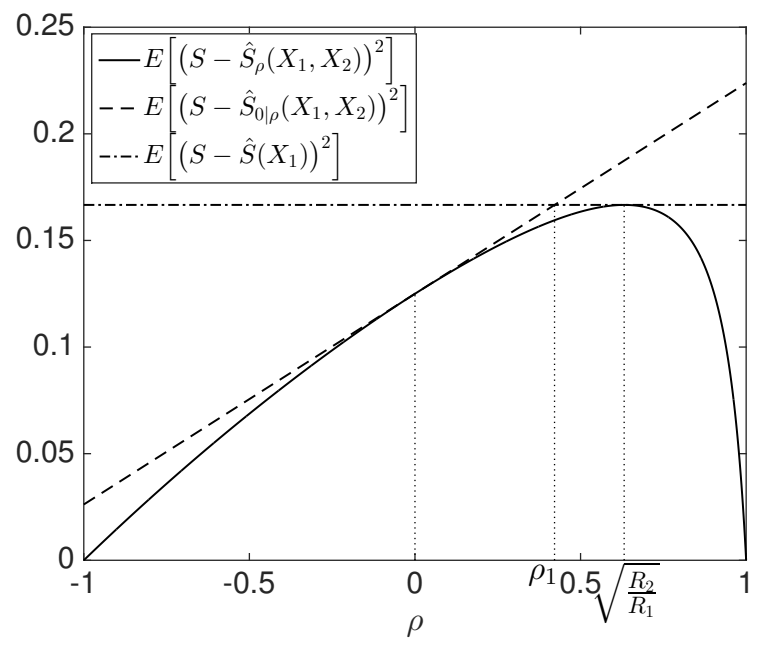

(b) Different SNRs

Fig. 3. Variation of the MSE as a function of $\rho$

$$
\hat{S}_{-1}\left(X_{1}, X_{2}\right)=\frac{\sqrt{R_{1}} X_{1}+\sqrt{R_{2}} X_{2}}{\sqrt{R_{1}}+\sqrt{R_{2}}} .
$$

Notice that, for $R_{1}=R_{2}$, the estimator (13) does not hold, because the two modalities are completely redundant (Fig. 2a). By contrast, for negatively-correlated noises, i.e $\rho \in[-1,0[$, we always manage to reduce the noise whatever the values of the signal to noise ratios $R_{1}$ and $R_{2}$. Accordingly, we always get better results than the independent and positively correlated cases (Fig. 2b). Thus, to get the most out of the modalities, the noise correlation has to be negative. Otherwise, the noises have to be strongly correlated, i.e $\left.\rho \in] \rho_{0}, 1\right]$, with different SNRs so that $\rho_{0} \rightarrow 0$.

We can observe in figures 2 and 3 that the results of $\mathrm{MI}$ and MSE, although giving different points of view, are consistent which is not surprising since the gradient of the MI is directly related to the MSE [5] in Gaussian channels.

\section{WRONG MODEL}

In this section we study the loss of information when we wrongly define the noise correlation coefficient. We consider that $\rho$ is unknown and denote by $\hat{\rho}$ the assumed noise correlation coefficient. To quantify the loss in information, we compare the information brought by the modalities considering the exact $\rho, I_{\rho}\left(S ;\left(X_{1}, X_{2}\right)\right)$, with the "information" brought by the modalities about $S$ when $\hat{\rho}$ is used instead of $\rho$, $I_{\hat{\rho} \mid \rho}\left(S ;\left(X_{1}, X_{2}\right)\right)$ (remember it is not a MI cf. SectionIII).

\section{A. $\hat{\rho}=0$}

Before investigating the loss for $\hat{\rho}$ in general, let us consider the case where $\hat{\rho}=0$. In this particular case the modalities are assumed to have independent noises while the noises are actually correlated. In the literature, the noises between data sets are commonly assumed to be independent for computation simplicity. If the noises are actually correlated the error induced by this assumption is given by (6). Using jointly Gaussian distributions, it follows:

$$
\begin{gathered}
I_{\rho}\left(S ;\left(X_{1}, X_{2}\right)\right)-I_{0 \mid \rho}\left(S ;\left(X_{1}, X_{2}\right)\right)=\rho \frac{\sqrt{R_{1} R_{2}}}{1+R_{1}+R_{2}} \\
+\frac{1}{2} \log \frac{\left(1+R_{1}\right)\left(1+R_{2}\right)-\left(\sqrt{R_{1} R_{2}}+\rho\right)^{2}}{\left(1-\rho^{2}\right)\left(1+R_{1}+R_{2}\right)} .
\end{gathered}
$$

For convenience, we denote this loss by $\Delta I(\rho, 0)$. The loss equals zero iff $\rho=0$. Eq. (15) shows that the loss becomes infinite for $\rho=1\left(R_{1} \neq R_{2}\right)$ and $\rho=-1$. This is because $S$ is not perfectly recovered with a wrong value $\hat{\rho}=0$, while it is with the exact one. In fact, for infinite MI an exact quantification of the loss is given by the MSE. The MSE of estimating $S$ using jointly $X_{1}$ and $X_{2}$ when the noises are assumed independent, is

$$
\begin{aligned}
& E\left[\left(S-\hat{S}_{0 \mid \rho}\left(X_{1}, X_{2}\right)\right)^{2}\right] \\
& \quad=\sigma^{2} \frac{1}{1+R_{1}+R_{2}}+2 \sigma^{2} \rho \frac{\sqrt{R_{1} R_{2}}}{\left(1+R_{1}+R_{2}\right)^{2}}
\end{aligned}
$$

The error (16) is a linear function of $\rho$, and is tangent at $\rho=0$ to the MSE of estimation using both modalities and exact noise correlation (Fig.3). Moreover, the error (16) intersects the MSE of estimation based uniquely of $X_{1}$ for $\rho=\rho_{1}$, where $\rho_{1}=\frac{1+R_{1}+R_{2}}{2\left(1+R_{2}\right)} \sqrt{\frac{R_{1}}{R_{2}}}$. Thus in the range $\left[\rho_{1}, 1\right]$, $X_{1}$ holds more information than does jointly $X_{1}$ and $X_{2}$ with the assumption of noises independence. Therefore, in this case using two modalities will give worse results for estimating $S$ than using $X_{1}$ alone. Notice that if $R_{2} \ll R_{1}, \rho_{1}$ tends towards zero. Furthermore, $\rho_{1}$ is never negative, which means that ignoring a negative correlation induces loss, but always insure more information than does the best (with the highest 
SNR) single modality. Finally, note that $I_{0 \mid \rho}\left(S ;\left(X_{1}, X_{2}\right)\right)$ never reaches $I\left(S ; X_{2}\right)$ : it means that the MI of the joint observation, with wrong assumption $\hat{\rho}=0$, is always better than using the worse single modality.

B. $\hat{\rho} \in[-1,1]$

In the general case, considering model (1) and assuming that $\hat{\rho} \neq \rho$ induces the following loss of information:

$$
\begin{aligned}
& \Delta I(\rho, \hat{\rho}) \\
& =\frac{1}{2} \log \frac{\left(1-\hat{\rho}^{2}\right)\left(\left(1+R_{1}\right)\left(1+R_{2}\right)-\left(\sqrt{R_{1} R_{2}}+\rho\right)^{2}\right)}{\left(1-\rho^{2}\right)\left(\left(1+R_{1}\right)\left(1+R_{2}\right)-\left(\sqrt{R_{1} R_{2}}+\hat{\rho}\right)^{2}\right)} \\
& +(\rho-\hat{\rho}) \frac{\left(\sqrt{R_{1}} \hat{\rho}-\sqrt{R_{2}}\right)\left(\sqrt{R_{2}} \hat{\rho}-\sqrt{R_{1}}\right)}{\left(1-\hat{\rho}^{2}\right)\left(\left(1+R_{1}\right)\left(1+R_{2}\right)-\left(\sqrt{R_{1} R_{2}}+\hat{\rho}\right)^{2}\right)} .
\end{aligned}
$$

The loss in (17) depends on the SNRs and the exact and wrong noise correlation coefficients, $\rho$ and $\hat{\rho}$ respectively. Since, from (6), $\Delta I(\rho, \hat{\rho})$ is the KL divergence between the exact and wrong densities for $S$ given $\left(X_{1}, X_{2}\right)$, it is always positive and equals zero iff $\hat{\rho}=\rho . \Delta I(\rho, \hat{\rho})$ increases with $|\rho-\hat{\rho}|$, since the mismatch between the exact and wrong densities for $S$ given $\left(X_{1}, X_{2}\right)$ increases with the difference $|\rho-\hat{\rho}|$. Eq. (17) shows that $\Delta I(\rho, \hat{\rho}) \rightarrow+\infty$ for $\rho=1\left(R_{1} \neq R_{2}\right)$ and $\rho=-1$, since the signal $S$ can be perfectly recovered considering the exact noise correlation $\left(I_{\rho}\left(S ;\left(X_{1}, X_{2}\right)\right) \rightarrow+\infty\right)$. Also, $\Delta I(\rho, \hat{\rho}) \rightarrow+\infty$ for $\hat{\rho}=1\left(R_{1} \neq R_{2}\right)$ and $\hat{\rho}=-1$, as we wrongly assume that the target signal could be perfectly recovered $\left(I_{\hat{\rho} \mid \rho}\left(S ;\left(X_{1}, X_{2}\right)\right) \rightarrow-\infty\right)$. In the case of infinite information loss, an exact quantification of the loss is given by the MSE. The MSE of estimating $S$ upon $X_{1}$ and $X_{2}$, considering the wrong noise correlation coefficient $\hat{\rho}$ is:

$$
\begin{aligned}
& E\left[\left(S-\hat{S}_{\hat{\rho} \mid \rho}\left(X_{1}, X_{2}\right)\right)^{2}\right] \\
& \quad=\sigma^{2} \frac{1-\hat{\rho}^{2}}{\left(1+R_{1}\right)\left(1+R_{2}\right)-\left(\sqrt{R_{1} R_{2}}+\hat{\rho}\right)^{2}} \\
& \quad+2 \sigma^{2}(\rho-\hat{\rho}) \frac{\left(\sqrt{R_{1}} \hat{\rho}-\sqrt{R_{2}}\right)\left(\sqrt{R_{2}} \hat{\rho}-\sqrt{R_{1}}\right)}{\left(\left(1+R_{1}\right)\left(1+R_{2}\right)-\left(\sqrt{R_{1} R_{2}}+\hat{\rho}\right)^{2}\right)^{2}} .
\end{aligned}
$$

To investigate when the loss of information due to assuming $\hat{\rho}$ drops the information below that of one single modality, we compare $I_{\hat{\rho} \mid \rho}\left(S ;\left(X_{1}, X_{2}\right)\right)$ with $I\left(S ; X_{i}\right)$ :

$$
\begin{aligned}
I_{\hat{\rho} \mid \rho}\left(S ;\left(X_{1}, X_{2}\right)\right) & -I\left(S ; X_{i}\right) \\
& =I_{\rho}\left(S ; X_{j} \mid X_{i}\right)-\Delta I(\rho, \hat{\rho}) .
\end{aligned}
$$

Eq. (19) shows that, $I_{\hat{\rho} \mid \rho}\left(S ;\left(X_{1}, X_{2}\right)\right)=I\left(S ; X_{i}\right)$ when the loss of information induced by considering $\hat{\rho} \neq \rho$ cancels the additional information conveyed by $X_{j}$. For model (1), the equality $I_{\hat{\rho} \mid \rho}\left(S ;\left(X_{1}, X_{2}\right)\right)=I\left(S ; X_{1}\right)$ holds in two cases:

1) If $\hat{\rho}=\sqrt{\frac{R_{2}}{R_{1}}}, \forall \rho$
With this assumption, we consider that the modality $X_{2}$ is redundant w.r.t $X_{1}$ and ignore all the additional information given by $X_{2}$. In fact the optimal estimator of $S$ using jointly $X_{1}$ and $X_{2}$ considering $\hat{\rho}, \hat{S}_{\hat{\rho} \mid \rho}\left(X_{1}, X_{2}\right)$ equals the optimal estimator of $S$ using $X_{1}$ alone, $\hat{S}\left(X_{1}\right)$. This case can not occur for the second modality, according to the assumption $R_{1} \geq R_{2}$.

2) If $\rho=\rho_{1}$ and $\hat{\rho} \neq \sqrt{\frac{R_{2}}{R_{1}}}$

where $\rho_{1}=\hat{\rho}+\frac{\left(\sqrt{R_{1}} \hat{\rho}-\sqrt{R_{2}}\right)\left(\left(1+R_{1}\right)\left(1+R_{2}\right)-\left(\sqrt{R_{1} R_{2}}+\hat{\rho}\right)^{2}\right)}{2\left(1+R_{1}\right)\left(\sqrt{R_{2}} \hat{\rho}-\sqrt{R_{1}}\right)}$. Unlike the first case, the optimal estimators using $X_{1}$ alone and using jointly $X_{1}$ and $X_{2}$ considering $\hat{\rho}$ are different i.e. $\hat{S}_{\hat{\rho} \mid \rho}\left(X_{1}, X_{2}\right) \neq \hat{S}\left(X_{1}\right)$. However, they have the same MSE i.e. $E\left[\left(S-\hat{S}_{\hat{\rho} \mid \rho}\left(X_{1}, X_{2}\right)\right)^{2}\right]=E\left[\left(S-\hat{S}\left(X_{1}\right)\right)^{2}\right]$. Which means that, $X_{1}$ alone and $\left(X_{1}, X_{2}\right)$ jointly considering $\hat{\rho}$ communicate the same amount of information but different piece of information.

Finally, it is beneficial to use the best single modality $X_{1}$ alone if $\rho<\rho_{1}$ and $\sqrt{\frac{R_{2}}{R_{1}}}<\hat{\rho}$ or if $\rho>\rho_{1}$ and $\sqrt{\frac{R_{2}}{R_{1}}}>$ $\hat{\rho}$ (since $I_{\hat{\rho} \mid \rho}<I\left(S ; X_{1}\right)$ ). Furthermore, note that even the worse single modality $X_{2}$ can convey more information than does jointly $X_{1}$ and $X_{2}$ considering $\hat{\rho}$, for $\rho>\rho_{2}$. where: $\rho_{2}=\hat{\rho}+\frac{\left(\sqrt{R_{2}} \hat{\rho}-\sqrt{R_{1}}\right)\left(\left(1+R_{1}\right)\left(1+R_{2}\right)-\left(\sqrt{R_{1} R_{2}}+\hat{\rho}\right)^{2}\right)}{2\left(1+R_{2}\right)\left(\sqrt{R_{1}} \hat{\rho}-\sqrt{R_{2}}\right)}$.

\section{CONCLUSION}

In this paper, we raised the question of how the links between modalities can improve or decrease the estimation accuracy and information content. We considered a simple model and investigated the role of noise correlation which represents the link between two modalities. Our results suggests that the noise correlation usually increases information and boosts the performance. Moreover, negative noise correlation is the key to always get the most out of both modalities. However, under the assumption of a wrong noise correlation it may be beneficial to consider a single modality than two modalities. Future research should consider other characteristics of multimodality and more complex models.

\section{REFERENCES}

[1] D. Lahat, T. Adali, and C. Jutten, "Multimodal data fusion: An overview of methods, challenges, and prospects," Proceedings of the IEEE, vol. 103, no. 9, pp. 1449-1477, 2015.

[2] F. Peng and B. Chen, "Decentralized estimation with correlated additive noise: Does dependency always imply redundancy?" in Signals, Systems and Computers, 2013 Asilomar Conference on, Nov 2013, pp. 677-681.

[3] T. M. Cover and J. A. Thomas, Elements of Information Theory. New York, NY, USA: Wiley-Interscience, 1991.

[4] S. M. Kay, Fundamentals of Statistical Signal Processing: Estimation Theory. Upper Saddle River, NJ, USA: Prentice-Hall, Inc., 1993.

[5] D. Palomar and S. Verdu, "Gradient of mutual information in linear vector gaussian channels," Information Theory, IEEE Transactions on, vol. 52, no. 1, pp. 141-154, Jan 2006.

[6] S. Nirenberg, S. M. Carcieri, A. L. Jacobs, and P. E. Latham, "Retinal ganglion cells act largely as independent encoders," Nature, vol. 411, 2001. [Online]. Available: http://dx.doi.org/10.1038/35079612

[7] D. Lahat, T. Adali, and C. Jutten, "Challenges in multimodal data fusion," in Signal Processing Conference (EUSIPCO), 2014 Proceedings of the 22nd European, Sept 2014, pp. 101-105. 